\section{Rapidly involuting congenital hemangioma associated with Kasabach-Merritt Syndrome}

\author{
Rosalia Ballona ${ }^{1 *}$, Jean Zevallos ${ }^{2}$ and Jeanette Núñez ${ }^{3}$ \\ ${ }^{1}$ Pediatric Dermatology Service, Department of Medicine, Instituto Nacional de Salud del Niño, Breña, \\ Lima, Perú \\ 2Dermatology Service, Department of Medicine, Hospital Nacional Dos de Mayo, Lima, Perú \\ ${ }^{3}$ Dermatology Service, Department of Medicine, Hospital Nacional Carlos Alberto Seguín Escobedo, \\ Arequipa, Perú
}

\section{Abstract}

Background: Rapidly involuting congenital hemangioma $(\mathrm{RICH})$ is a rare vascular tumor that is present at birth and involutes during the first year of life. Kasabach-Merritt syndrome (KMS) is a complication of some vascular tumors such as kaposiform hemangioendothelioma and tufted angioma associated with thrombocytopenia and coagulopathy.

Results: The case of a 2-month-old infant with a diagnosis of $\mathrm{RICH}$ with thrombocytopenia and coagulation disorder, successfully treated with surgical excision without complications or recurrence is presented.

Conclusion: The association between RICH and KMS is rare. Histopathological study, immunohistochemistry and ultrasound findings are important for the diagnosis.

Brief summary: This report covers the rare association between rapidly involuting congenital hemangioma and Kasabach-Merritt syndrome in a 2-months-old female infant.

\begin{abstract}
More Information
*Address for Correspondence: Rosalia Ballona, Pediatric Dermatology Service, Instituto Nacional de Salud del Niño, Lima-Peru. Av. Brasil 600, Breña 15083, Peru, Tel: +51999352357 .

Email: rballona@gmail.com

Submitted: April 26, 2021

Approved: May 14, 2021

Published: May 17, 2021

How to cite this article: Ballona R, Zevallos $\mathrm{J}$, Núñez J. Rapidly involuting congenital hemangioma associated with Kasabach-Merritt Syndrome. J Adv Pediatr Child Health. 2021; 4: 050-052.

DOI: 10.29328/journal.japch.1001032

Copyright: @ 2021 Ballona R, et al. This is an open access article distributed under the Creative Commons Attribution License, which permits unrestricted use, distribution, and reproduction in any medium, provided the original work is properly cited.

Keywords: Rapidly involuting congenital hemangioma; Kasabach-Merritt Syndrome

Abbreviations: RICH: Rapidly Involuting Congenital Hemangioma; KMS: KasabachMerritt Syndrome; KHE; Kaposiform Hemangioendothelioma: TA: Tufted Angioma; $\mathrm{CH}$ : Congenital Hemangioma; $\mathrm{NICH}$ : NonInvoluting Congenital Hemangioma: $\mathrm{PICH}$ : Partially Involuting Congenital Hemangioma; MRI: Magnetic Resonance Imaging
\end{abstract}

Check for updates

OPEN ACCESS

\section{Introduction}

Congenital hemangioma $(\mathrm{CH})$ is a rare vascular tumor that has reached its full size at birth [1]. It has been classified into three subtypes: rapidly involuting congenital hemangioma (RICH), non-involuting congenital hemangioma (NICH) and partially involuting congenital hemangioma (PICH) [2]. CHs rarely present complications, such as ulceration and bleeding [3]. Kasabach-Merritt syndrome (KMS) is a severe complication of some vascular tumors, which associates profound thrombocytopenia and coagulopathy that can be lifethreatening [4]. Tumors associated with KMS are kaposiform hemangioendothelioma (KHE) and tufted angioma (TA) $[4,5]$. We present a male infant with RICH and KMS with complete resolution after surgical treatment.

\section{Case report}

A 2-month-old female infant presented to the emergency department with a two-week history of hypotonia, poor feeding and lethargy. His medical history included a term dystocic delivery due to a congenital mass on the left thigh and a hospitalization for anemia one week after birth. His physical examination revealed a soft violaceous mass of $13 \times 12 \mathrm{~cm}$ in the posterior left thigh surrounded by a pale halo (Figure 1). No other lesions were found.

On admission, a complete blood count revealed a platelet count of $48000 / \mathrm{mm} 3$, neutrophil count of $600 / \mathrm{mm} 3$ and a hemoglobin of $5.9 \mathrm{~g} / \mathrm{dL}$. Coagulation test results showed a prothrombin time of 20.7 seconds, an activated partial thromboplastin time of 53.9 seconds, a fibrinogen level of 66.2 $\mathrm{mg} / \mathrm{dL}$ and a D-dimer level of $35.5 \mathrm{ng} / \mathrm{dL}$. Patient was treated with prednisone, vincristine and received packed red blood cells and platelet transfusion with persistence of anemia and thrombocytopenia. A Doppler ultrasound showed a mass with high flow and venous lakes. A magnetic resonance imaging (MRI) examination was subsequently performed. MRI with contrast revealed feeding vessels from the femoral vein and collateral circulation of the femoral artery. 
A diagnosis of RICH associated with KMS was made. Due to poor response to medical treatment, surgical excision was performed at 3 months of age. In the surgery intervention, tumor tissue was dissected until the origin of both feeding vessels were found. Then, they were ligated and the tumor was excised.

The histopathological examination showed the presence of lobules containing small capillaries surrounded by fibrosis tissue and hemosiderin depositions (Figure 2). Immunohistochemical staining for Glut-1 was negative and positive for CD31 (Figures 3,4). Postoperative evolution was good, without complications or recurrence up to 6 months after the intervention.

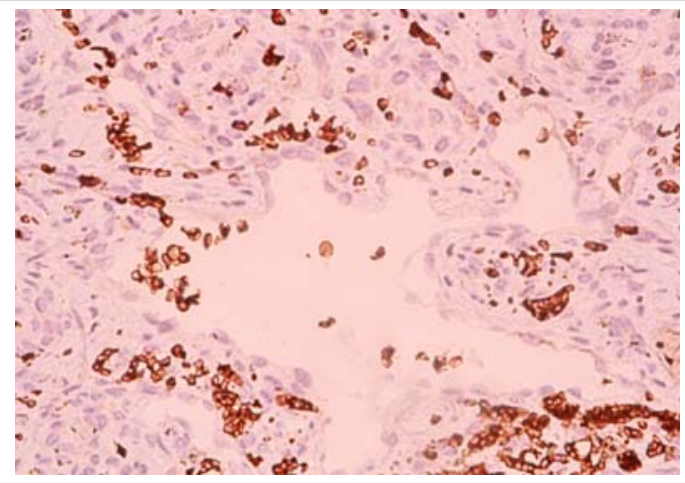

Figure 1: Violaceous mass with peripheral pale halo on the left thigh.

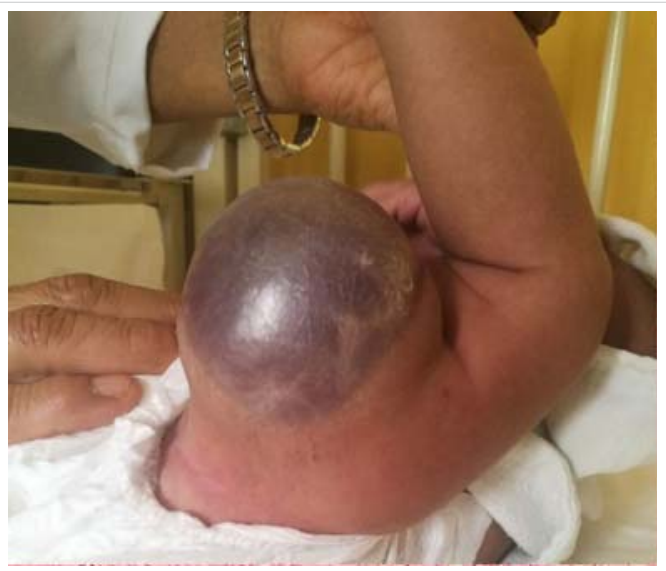

Figure 2: Lobules containing capillaries surrounded by fibrosis and hemosiderin depositions.

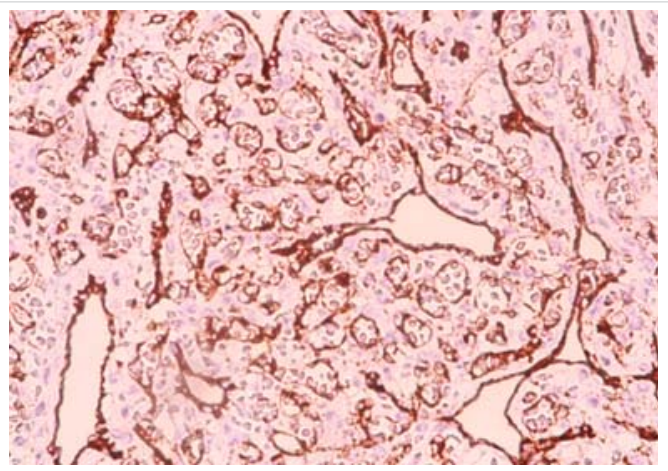

Figure 3: Capillaries with negative staining for Glut-1.

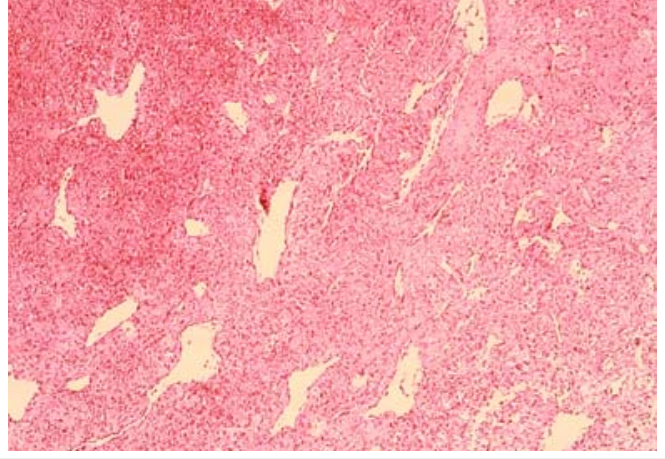

Figure 4: Capillaries with positive staining for $\mathrm{CD} 31$ in areas of fibrosis.

\section{Discussion}

$\mathrm{CH}$ is a rare vascular tumor that represents $3 \%$ of all hemangiomas. CHs commonly are solitary tumors, affect head/neck and extremities and do not tend to show a gender predilection [1]. RICH involutes in the first 6-14 months of life [1]. These tumors can rarely cause complications, such as ulceration and bleeding [3]. As well, RICH associated with transient thrombocytopenia and coagulation abnormalities have been reported during the first week of life [5]. KMS is a complication of vascular tumors such as KHE and TA, characterized by the presence of thrombocytopenia and coagulation disorders, sometimes associated with anemia and elevated D-dimer levels [4,5]. There are three case reports of $\mathrm{CHs}$ associated with coagulation disorders considered as KMS. Boon, et al. [6] reported two cases of RICH associated with coagulopathy. However, more details were not provided [6]. In the case reported by Hsiao, et al. [7], the lowest platelet count was $107000 / \mathrm{mm} 3$ during the first days of life and the histopathology results are not detailed. Hosono, et al. [8] reported a patient with a large $\mathrm{CH}$ with a platelet count of 54000 at day 2, which normalized around 2 weeks of life after a coil embolization treatment at day 3. These cases could be considered as $\mathrm{CHs}$ associated with transient thrombocytopenia since the platelet count is in the range of $5,000-62,000 / \mathrm{mm} 3[5,9]$.

RICHs have a variable morphology, they present commonly as reddish violaceus tumors with peripheral pale halo, tiny or coarse telangiectasias and sometimes with central ulceration, linear scar and central nodularity [10]. Clinically, tumors complicated by KMS increase in size, change in colour, become firm and often develop petechiae locally and elsewhere [5]. There are ultrasounds features only seen in $\mathrm{CHs}$, such as venous lake, venous ectasia and calcification. Waelti, et al. [11] suggest that venous ectasia and venous lakes could be associated with bleeding or heart failure, so these patients should be monitored more closely. Histopathologically, RICH is characterized by well-defined, variably sized lobules containing small capillaries and prominent draining vessels in the dermis and subcutaneous tissue. In the early stages, blood vessels possess a thin basement membrane and the endothelial is moderately plump. Subsequently, the basement 
membrane thickens and the lobes are surrounded by fibrosis, calcification, thrombosis and hemosiderin deposition [1,5]. All CHs are negative for Glut-1 immunostaining.

Generally, treatment in uncomplicated CHs is not necessary [12], unlike complicated $\mathrm{CHs}$ by ulceration, bleeding or hemodynamic instability, treatment with embolization or excision of the tumor may be chosen $[9,12]$. In the reported cases of CHs associated with transient thrombocytopenia, treatments included systemic corticosteroids therapy, platelet transfusions and embolization [5,9]. Management of KMS associated with KHE or TA includes supportive care, pharmacologic and surgery treatment. Supportive care with platelet transfusions, cryoprecipitate and packed red blood cells should only be performed if there is active bleeding or severe symptomatic anemia. Pharmacologic management is often first line and can achieve hemostatic stability. There have been reported many pharmacologic therapies involving steroids, vincristine, interferon-alfa, antiplatelet agents, propranolol and sirolimus with variable outcomes $[4,13]$. Surgical treatment should be performed as soon as possible after the correction the coagulation disorders to prevent exacerbation of thrombocytopenia and coagulopathy. Our patient was refractory to medical treatment with prednisone and vincristine, requiring surgical treatment, without complications or tumor recurrence up to 6 months after the intervention.

\section{Conclusion}

The association of RICH with SKM is rare and represent a diagnostic and treatment challenge. Histopathological study and a negative Glut-1 immunostaining are required for diagnosis. Also, ultrasound findings could guide the diagnosis. Although the tumor excision was a high-risk intervention, it was resolutive and did no associate any further complications in our patient.

\section{References}

1. Bras $S$, Mendes-Bastos $P$, Amaro $C$. Rapidly involuting congenital hemangioma. An Bras Dermatol. 2017; 92: 861-863.

PubMed: https://pubmed.ncbi.nlm.nih.gov/29364450/
2. Mulliken JB, Enjolras O. Congenital hemangiomas and infantile hemangioma: missing links. J Am Acad Dermatol. 2004; 50: 875-882. PubMed: https://pubmed.ncbi.nIm.nih.gov/15153887/

3. Al Malki A, Al Bluwi S, Malloizel-Delaunay J, Mazereeuw-Hautier J. Massive hemorrhage: a rare complication of rapidly involuting congenital hemangioma. Pediatr Dermatol. 2018; 35: e159-e160. PubMed: https://pubmed.ncbi.nlm.nih.gov/29572940/

4. Mahajan P, Margolin J, lacobas I. Kasabach-merritt phenomenon: classic presentation and management options. Clin Med Insights Blood Disord. 2017; 10: 1179545×17699849.

PubMed: https://pubmed.ncbi.nlm.nih.gov/28579853/

5. Baselga E, Cordisco MR, Garzon M, Lee MT, Alomar A, et al. Rapidly involuting congenital haemangioma associated with transient thrombocytopenia and coagulopathy: a case series. $\mathrm{Br} \mathrm{J}$ Dermatol. 2008; 158: 1363-1370.

PubMed: https://pubmed.ncbi.nIm.nih.gov/18410425/

6. Boon LM, Enjolras O, Mulliken JB. Congenital hemangioma: evidence of accelerated involution. J Pediatr. 1996; 128: 329-335.

PubMed: https://pubmed.ncbi.nlm.nih.gov/8774499/

7. Hsiao $\mathrm{CH}$, Tsao PN, Hsieh WS, Chou HC. Huge, alarming congenital hemangioma of the scalp presenting as heart failure and KasabachMerritt syndrome: a case report. Eur J Pediatr. 2007; 166: 619-620. PubMed: https://pubmed.ncbi.nlm.nih.gov/16937128/

8. Hosono S, Ohno T, Kimoto H, Nagoshi R, Shimizu M, et al. Successful transcutaneous arterial embolization of a giant hemangioma associated with high-output cardiac failure and Kasabach-Merritt syndrome in a neonate: a case report. J Perinat Med. 1999; 27: 399-403. PubMed: https://pubmed.ncbi.nlm.nih.gov/10642962/

9. Larralde M, Solé J, Luna P, Mosquera T, Abad M. Hemangiomas congénitos rápidamente involutivos. Serie de 25 casos. Arch Argent Pediatr. 2014; 112: e61-e65.

10. Berenguer B, Mulliken JB, Enjolras $O$, et al. Rapidly involuting congenital hemangioma: clinical and histopathologic features. Pediatr Dev Pathol. 2003; 6: 495-510.

PubMed: https://pubmed.ncbi.nlm.nih.gov/15018449/

11. Waelti SL, Rypens F, Damphousse A, et al. Ultrasound findings in rapidly involuting congenital hemangioma $(\mathrm{RICH})$ - beware of venous ectasia and venous lakes. Pediatr Radiol. 2018; 48: 586-593. PubMed: https://pubmed.ncbi.nlm.nih.gov/29362838/

12. Rangwala S, Wysong A, Tollefson MM, Khuu P, Benjamin LT, et al. Rapidly involuting congenital hemangioma associated with profound, transient thrombocytopenia. Pediatr Dermatol. 2014; 31: 402-404. PubMed: https://pubmed.ncbi.nlm.nih.gov/22937785/

13. Tlougan BE, Lee MT, Drolet BA, Frieden IJ, Adams DM, et al. Medical management of tumors associated with Kasabach-Merritt phenomenon: an expert survey. J Pediatr Hematol Oncol. 2013; 35: 618-622. PubMed: https://pubmed.ncbi.nlm.nih.gov/23774156/ 